\title{
WALDBRÄNDE IN KANADA \\ UND IHRE BEKÄMPFUNG
}

\author{
HANS BERNHARD
}

Vor der Ankunft des weißen Mannes erstreckte sich der kanadische Wald in einem ununterbrochenen, bis zu zweitausend Kilometer breiten Gürtel von Küste zu Küste. Nur im trockenen Süden der drei Prärieprovinzen Manitoba, Saskatchewan und Alberta unterbrachen ausgedehnte Rasenflächen die Waldregion, und im nördlichen Landesteil sowie in den höhern Regionen des westlichen Gebirgslandes ging der Wald infolge zunehmender Kälte in die Tundra über.

Für die weißen Siedler bedeutete der Wald vorerst ein großes Hindernis. In mühsamer Arbeit schufen sie Platz für Siedlung und Anbau. Sie rodeten mit Hilfe des Feuers, zündeten das trockene Unterholz und einige gefällte Bäume an und entfernten die verkohlten Strünke.

Immer mehr aber erkannte der Mensch den großen und vielseitigen Wert des Waldes: für Bau- und Brennholz, später für die Herstellung von Zellulose und Papier, ebenso für die Regulierung des Wasserhaushaltes, für die Verminderung der Wassererosion, als Obdach und Nahrung für die Wildtiere und in unseren Tagen als beliebtes Ausflugsziel für Wanderer und Automobilisten.

Über zwei Milliarden Schweizer Franken beträgt allein die industrielle Ausnützung des kanadischen Waldes im Laufe eines Jahres, und man wird sich mit Recht fragen, ob Kanada auch schon zum Raubbau nach dem Vorbild europäischer Länder übergegangen sei. Diese Gefahr besteht aber noch nicht; denn die rund hundert Millionen Kubikmeter Holzabgang pro Jahr werden durch Neuwuchs mehr als nur ersetzt. Noch sind ein Drittel der ganzen Landesfläche mit Wald bestanden, natürlich Wald verschiedener Art und auch verschiedener Qualität. Etwa 70\% des produktiven Waldes sind im Besitze der Provinzregierungen und des Staates ${ }^{1}$, gehören also dem Volk.

Durch strenge Bestimmungen fordern die verschiedenen Regierungen die Wiederaufforstung, die gewöhnlich schon mit der Lizenz für den Holzschlag einbedungen wird.

Soweit wäre alles auf bestem Wege, um den wertvollen Wald auch späteren Generationen zu erhalten. Was zur Beunruhigung zwingt, ist einzig derjenige Teil des Waldes, der alljährlich durch Schädlinge und Waldbrände nutzlos verlorengeht. Man spricht von total $25 \%$ des Holzabganges. Natürlich ist die Zerstörung durch pflanzliche und tierische Schädlinge nicht so gut erfaßbar, sterben doch die Bäume oft erst im Laufe von Jahren ab. Diese Sorge soll uns hier aber nicht weiter beschäftigen. Festgestellt sei nur, daß man in Kanada diese Schädlinge mit allen zur Verfügung stehenden Mitteln bekämpft, immer mehr mit zugeführten Parasiten und Viren und durch Streuung von Insektiziden aus der Luft.

Viel besser registrierbar sind die Waldbrände, sowohl nach ihrer Häufigkeit, regionalen Verteilung und nach dem Ausmaß der angerichteten Verheerung. Natürlich bestehen große Unterschiede von Jahr zu Jahr, so daß wir in beiliegender Tabelle Mittelwerte für die Zeit von 1950-59 zusammengestellt haben (Tabelle) ${ }^{2}$. Danach zerstören jährlich 5650 Brände 837000 Hektaren Wald (gesamte Waldfläche der Schweiz 955541 Hektaren). Die starke Abweichung von solchen Mittelwerten zeigte besonders

1 Der Staat verfügt über den Baumbestand im Yukon- und Nordwest-Territorium (die von der Zentralregierung in Ottawa verwaltet werden), in den Nationalparks, den Indianerreservaten, den militärischen Anlagen sowie in den staatlichen Forstuntersuchungsgebieten.

2 Die Prinz-Eduard-Insel fehlt leider in dieser Zusammenstellung. Für 1960 verzeichnete diese Provinz 25 Waldbrände, 7300 Hektaren verwüstetes Waldland mit 221 ooo Dollar Schaden. Ausgaben für Brandbekämpfung sind nicht bekannt. 
das Jahr 1960 mit 8881 Bränden und einer viermal so großen Brandfläche, wie im Tabellenmittel angegeben. Im Rekordjahr 1961 wurden sogar 31/2 Millionen Hektaren (entsprechend der doppelten Fläche des Kantons Zürich) Wald vernichtet. Es ist zu erwarten, daß die drei Provinzen mit der größten Waldfläche, nämlich Quebec, Ontario und British Columbia, auch am meisten Waldbrände zu verzeichnen haben, wobei aber British Columbia sowohl nach der Zahl der Brände wie auch nach dem absoluten und prozentualen Ausmaß der zerstörten Fläche stark dominiert. Der erlittene Brandschaden ${ }^{3}$ sowie die Ausgaben für die Brandbekämpfung (was in der Statistik leider nicht auseinandergehalten wird), sind fast so groß wie für alle übrigen Provinzen und Territorien zusammen.

Warum entstehen Waldbrände, auf welche Ursachen gehen sie zurück? Zum besseren Vergleich benützen wir wieder die Mittelwerte aus dem Jahrzehnt 1950-59: $18 \%$ durch Raucher, 15\% durch Campieren, 14\% durch Funkenwurf von Lokomotiven, $10 \%$ durch Siedler, $5 \%$ durch industrielle Operationen, 3\% durch Böswilligkeit, $2 \%$ durch öffentliche Arbeiten, $10 \%$ durch verschiedene bekannte Ursachen, $4 \%$ durch verschiedene unbekannte Ursachen, $19 \%$ durch Blitz.

Somit fallen rund $80 \%$ aller Brände auf das Konto des Menschen, der abgesehen von der wirtschaftlichen Nutzung auch so als größter Feind des Waldes in Erscheinung tritt. Wie manches weggeworfene Streichholz, wie manche ungelöschte Zigarette und wieviele verlassene Feuerstellen haben schon zu einem Waldbrand geführt! Auch die Lokomotiven, die kilometerlange Waldschneisen durchfahren, können nicht gefährlich genug eingeschätzt werden. $\mathrm{Zu}$ den verschiedenen bekannten Ursachen gehören vor allem Abstürze von Flugzeugen, Autounfälle und Bruch elektrischer Leitungen. Nur von $4 \%$ aller Waldbrände ist die Ursache nicht bekannt, ein Beweis dafür, wie aufmerksam der Staat diese Gefahr verfolgt.

\begin{tabular}{|c|c|c|c|c|c|}
\hline $\begin{array}{l}\text { Mittelwerte } \\
1950-1959\end{array}$ & 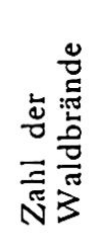 & 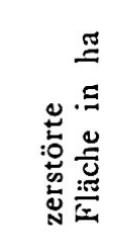 & 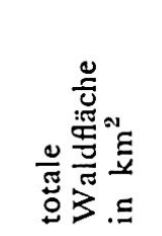 & 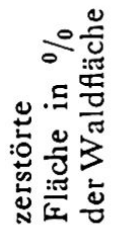 & 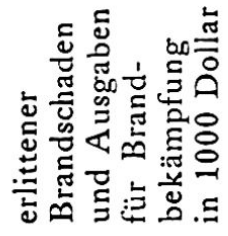 \\
\hline Neufundland & 176 & 7772 & 227381 & 0,0034 & 410 \\
\hline Neuschottland & 285 & 2595 & 42448 & 0,006 & 264 \\
\hline Neubraunschweig & 221 & 3542 & $63 \circ 12$ & 0,0056 & 488 \\
\hline Quebec & 900 & 76276 & 979.362 & 0,0078 & 4283 \\
\hline Ontario & 1279 & 38440 & 677925 & 0,0057 & 1129 \\
\hline Manitoba & 259 & 85014 & 319360 & 0,027 & 997 \\
\hline Saskatchewan & 145 & 28419 & 304941 & 0,009 & 1745 \\
\hline Alberta & 233 & 115834 & 411976 & 0,028 & 798 \\
\hline $\begin{array}{l}\text { Britisch-Kolumbien } \\
\text { Bundesgebiete }\end{array}$ & 1937 & 175626 & 693182 & 0,025 & 9100 \\
\hline Yukon & 60 & 94622 & 210308 & 0,045 & 32 \\
\hline Nordwestterritorium & 65 & 205196 & 504014 & 0,041 & 175 \\
\hline Nationalparks & 31 & 980 & - & - & 116 \\
\hline Indianerreservate & 55 & $29 \mathrm{o} 2$ & - & - & 75 \\
\hline Militärische Anlagen & 4 & 2 & - & - & 936 \\
\hline Kanada & 5650 & 837221 & 4436328 & 0,019 & 2o 548 \\
\hline
\end{tabular}

Noch am Anfang unseres Jahrhunderts war die Waldbrandbekämpfung in Kanada nicht über bescheidene Anfänge hinausgekommen. Heute bestehen in allen Provinzen Gesetze zur Bestrafung nachläßiger und unachtsamer Verursachung von Waldbränden, vor allem aber modernste Einrichtungen zur Entdeckung und Bekämpfung derselben.

3 Kann natürlich nur annähernd erfaßt werden, ohne Jungwald, Bodenzerstörung und Begleitschäden wie Vermehrung der Erosion usw. 
Es ist klar, daß ein Waldbrand nur in seinen Anfängen mit Erfolg bekämpft werden kann. Wenn er einmal eine gewisse Größe erreicht hat, entwickelt sich in einem solchen Brandherd eine große Hitze. Lebende, grüne Bäume brennen, als ob sie aus Papier wären. Brennende Holzstücke werden durch die heiße Luft und den Wind oft kilometerweit weggetragen und entfachen immer wieder neue Brandherde. Das haben schon die früheren Siedler erfahren, als sie gelegte Brände nicht mehr unter Kontrolle halten konnten. So fielen dem ältest erwähnten Brande vom Jahre 1825 im Miramichital in Neubraunschweig verschiedene Städte und Dörfer, 160 Menschenleben und die gesamte Viehhabe vieler Farmer zum Opfer. Der Brand wütete viele Tage, das Riesenfeuer war 40 Kilometer breit und fraß sich 120 Kilometer vorwärts. Aber auch in . unserem Jahrhundert sind durch Waldbrände wiederholt Menschen umgekommen, so bei der Katastrophe von Ontario 1916 mindestens 224 Personen. Da der Brand alle Spuren ausgelöscht hatte, ist ihre genaue Zahl nie bekannt geworden. Einmal kommt der Brand schließlich doch zum Stillstand, vielleicht wenn der Wind dreht, kräftiger Regen einsetzt oder an einem großen Fluß.

Wie trostlos ist ein abgebrannter Wald, auch in der unbesiedelten Region! Um die verkohlten Strünke größerer Bäume breitet sich der schwarze Waldboden. Alles Kleintier ist gestorben, und in den Randgebieten finden sich partiell verbrannte, eingegangene Tiere. Erst nach Jahren ist der Jungwald wieder so hoch, daß sich die Brandlücke allmählich schließt.

Da nur kleine Waldbrände erfolgreich bekämpft werden können, ist ihre frühzeitige Entdeckung von größter Bedeutung, und die neuzeitliche Brandbekämpfung geht denn auch in dieser Richtung. Grundlage für jede Übermittlung eines entdeckten Brandherdes ist eine genaue Karte des gesamten Waldgebietes. Mit Hilfe von Luftaufnahmen verfertigte Kanada die besten diesbezüglichen Karten der Welt. Darauf sind die Standorte der vielen Beobachtungstürme angegeben. Diese meist mit einer Kabine versehenen Stahlgerippe sind in besonders gefährdeten Gebieten errichtet worden. Saskatchewan besitzt 69 solcher Beobachtungsstationen. Die Richtung des Brandes wird mit dem sogenannten Feuersucher festgestellt, die Entfernung geschätzt. Kann ein Brand gleichzeitig von zwei Beobachtungszentren festgestellt werden, liegt er im Schnittpunkt beider Richtungsangaben. Festgestellte Brände werden sofort durch Radioverbindung weitergeleitet. Natürlich genügen diese Beobachtungstürme, und wären sie noch viel zahlreicher, bei weitem nicht. Zudem sind sie oft durch den Rauch benachbarter Brände in ihrer Aufgabe behindert. Deshalb kommt noch die Beobachtung durch Straßenpatrouillen, Eisenbahnen und besonders diejenige aus der Luft hinzu. Saskatchewan, das mit rund 300000 Hektaren Wald erst an 7. Stelle der Provinzen und Territorien steht, verfügt über ein Netz von 850 Radiostationen zur Übermittlung von Bränden. Quebec, Ontario, Manitoba, Saskatchewan und Alberta haben ihren eigenen Flugdienst für dieses Ressort, während in anderen Provinzen kommerzielle Unternehmen einspringen.

Glücklicherweise ist die Waldbrandgefahr nur während weniger Wochen eines Jahres akut. Die meisten Feuersbrünste fallen in die Zeit von Mai bis August.

Im Zeitraum 1950—59 verteilten sich die Waldbrände folgendermaßen über das Jahr:

$\begin{array}{lllllrrrrrrrr} & 1 & 2 & 3 & 4 & 5 & 6 & 7 & 8 & 9 & 10 & 11 & 12 \\ \text { Provinzen } & \circ & \circ & 1 & 8 & 22 & 13 & 23 & 21 & 7 & 4 & 1 & \circ \\ \text { Yukon } & \circ & \circ & 0 & 3 & 13 & 34 & 20 & 18 & 8 & 2 & 0 & 2 \\ \text { NW-Territorium } & 0 & 0 & 0 & 1 & 7 & 29 & 41 & 20 & 1 & 1 & 0 & 0\end{array}$

So vernichteten z. B. im Jahre 1961 in Ontario 1305 Feuersbrünste 480000 Hektaren Wald (entsprechend der Fläche des Kantons Obwalden), fast alle im Nordwesten des Landes, zwischen Mitte Juni und Mitte Juli. Und die Ursachen? Extreme Trok- 
kenheit wegen Regenmangels und wenig Schneefa!l im vorangegangenen Winter. Gewitter hatten $34 \%$ aller Brände mit $90 \%$ der zerstörten Fläche verursacht. Es ist also wichtig zu wissen, bei welchen Wetterlagen und Bodenzuständen und in welchen Gebieten Feuer zu erwarten sind, um rechtzeitig disponieren zu können. In Saskatchewan wird während der größten Gefahrenperiode eine Gruppe von Fallschirmspringern ständig in Alarmbereitschaft gehalten.

Festgestellte Waldbrände werden sofort per Radio oder Telephon zum ForstHauptquartier weitergeleitet und dort auf einer Karte eingetragen. Die weitere Disposition richtet sich weitgehend nach der Abgelegenheit und Zugänglichkeit des Brandherdes. Je nachdem wird man auf dem Land- oder Wasserweg, immer mehr aber mit Flugzeugen und Helikoptern, anrücken. Verschiedene Provinzen haben zu diesem Zweck kleine Luftbasen errichtet, so z. B. Lac du Bonnet, Norway House, Thicket Portage u. a., in Manitoba. Oft übernehmen solche Equipen nur die erste Brandbekämpfung, bis weitere Verstärkung eintrifft. Ist der Brandherd klein oder kein Wasser in der Nähe, so wird alles brennbare Material in breitem Umkreis niedergelegt. Steht hingegen Wasser zur Verfügung, so werden Handpumpen aus mitgetragenen Wassertanks oder dann längere Schlauchleitungen eingesetzt. Nicht immer sind solche Unternehmen von Erfolg gekrönt. Aber sie haben schon manchen Brand gelöscht und die aufgewendeten Mittel um ein Vielfaches eingebracht. Daß besonders in abgelegenen, dünnbesiedelten Gebieten die Waldbrände größere Ausmaße erreichen, zeigen die beiden Zahlen von Yukon-Distrikt und Nordwest-Territorium, trotzdem gerade hier die Brandbekämpfung wegen der geringen Baumdichte verhältnismäßig leicht wäre.

Die Brandbekämpfung in den Wäldern Kanadas ist heute zu einer Routinesache geworden. Schon studiert man den Einsatz von Chemikalien aus der Luft und weitere Verbesserungen zur Branderuierung und -bekämpfung.

Ebenso wichtig und viel primärer ist die Verhütung der Waldbrände. Da rund $80 \%$ durch den Menschen verursacht werden, ist deren Verminderung absolut möglich. Wir haben bereits die verschiedenen Ursachen, die zwischen 1950 und 1959 zu Waldbränden führten, festgehalten. Wo kann Abhilfe geschafft werden? Da wäre einmal der Funkenwurf der Lokomotiven, der ein Siebentel aller Brände entfacht. Es sind unendlich lange Strecken, im Osten und Westen des Landes, wo die Bahntrassees in Waldschneisen liegen, und in nächster Zukunft werden die Lokomotiven in Kanada zum Teil immer noch mit Dampf betrieben werden. Man hat aber an ihren Kaminen besondere Funkenfänger eingebaut, die Waldschneisen wesentlich verbreitert und den Beobachtungsdienst in diesen Zonen stark ausgebaut.

Seit der Schaffung der Reservate sind die gefährlichen Lagerfeuer der Indianer immer seltener geworden, laut Tabelle nur noch 55 kleinere Brände im Jahr. Dafür ist der weiße Autofahrer und Tourist an ihre Stelle getreten. Der Staat ist bestrebt, diesen Leuten den Aufenthalt im Wald so angenehm wie möglich zu machen und hat zu diesem $Z_{\text {weck }}$ Blockhäuser, Sitzgelegenheiten und feste Feuerstellen errichtet. Das genügt natürlich nur für einen kleinen Teil der Besucher. Die anderen campieren frei und besonders im Sommer zur gefährdeten Zeit. So ist zum Beispiel während der Kriegsjahre die Zahl der Brände stark zurückgegangen. Das Benzin war damals rationiert und der Touristenverkehr stark eingeschränkt. Um auch diese Leute, die als Raucher und Campierer rund ein Drittel aller Brände verursachen, auf die drohende Gefahr aufmerksam zu machen, appelliert der kanadische Forstdienst an groß und klein durch leuchtend gelbe Tafeln, die überall längs waldgesäumter Straßen oder bei Campingplätzen angebracht sind und die Aufschrift tragen: Prevent Forest Fires, it Pays!

Noch kompensiert der große Waldnachwuchs nicht nur die ökonomische Nutzung des Waldes, sondern auch seine Zerstörung durch Waldbrände und Schädlinge. Die weitsichtige und umfassende Planung des kanadischen Forstdienstes wird sich aber immer mehr auch auf dem Sektor der Waldbrandbekämpfung bezahlt machen. 


\title{
INCENDIES FORESTIERS AU CANADA
}

Autrefois, la forêt canadienne rendait difficile la colonisation par la race blanche. La forêt s'intègre aujourd'hui au loin dans le processus économique et se représente d'une importance multiple et d'une valeur inestimable. Malgré d'une forte exploitation, la diminution annuelle du bois sera suppléée par une forte croissance. Une partie importante du bois abatagé (env. 25\%) est ravagé encore toujours par des parasites et par des incendies forestiers. Ces incendies se voient provoquer par des causes diverses, mais surtout par l'inattention et par la négligence des hommes (env. 80\%). Un bon système, fonctionnant pour la découverte immédiate et pour la lutte contre les incendies forestiers ( $\mathrm{par}$ an plus que 5000) comme par des mesures appliquées, nous laisse attendre que les provinces canadiennes pourront diminuer toujours plus ces grandes pertes inutiles du bois.

\section{EIN BESUCH CARL RITTERS BEI JAKOB MELCHIOR ZIEGLER IN WINTERTHUR}

\author{
EDUARD IMHOF
}

Im Jahre 1959 waren es hundert Jahre her seit dem Tode Carl Ritters. Seine unvergänglichen Verdienste um die erdkundliche Forschung wurden durch Gedenkfeiern und Veröffentlichungen gewürdigt. Es ist nicht unsere Absicht, solchen Ehrungen hier weitere beizufügen oder gar Neues zur geographischen Forschungsgeschichte beizutragen. Da aber Carl Ritter sehr enge Beziehungen zur Schweiz pflegte, möchten wir seine ebenso anziehende wie sympathische Schilderung eines Besuches bei Jakob Melchior Ziegler in Winterthur und eines Ausfluges nach Zürich und in die Zentralschweiz der Vergessenheit entreißen. Diese Schilderung findet sich in einem Briefe, den der Gelehrte im Jahre 1847 an seinen Bruder Johannes geschrieben hatte. Er ist abgedruckt in Lit. Nr. 1 und 2.

Die beiden Hauptakteure des Geschehens, Ritter und Ziegler, seien indessen dem weniger orientierten Leser kurz vorgesteilt.

Carl Ritter, geboren 1779 zu Quedlinburg, Deutschland, ist in Geographenkreisen berühmt als der Begründer einer wissenschaftlich fundierten Lehre der Geographie. An die Stelle früheren Aneinanderreihens statistischer Daten setzte er eine vergleichende Länder- und Erdkunde, arbeitete überall das Typische heraus und schilderte die kausalen Verflechtungen und historischen Wandlungen der Dinge.

Aus einer Arztfamilie stammend und früh verwaist, hatte sich der junge Mann einem vielseitigen Universitätsstudium zugewendet, wirkte hierauf als Hauslehrer in Frankfurt, Genf und in Italien, erarbeitete sich durch Selbststudium und auf vielen Reisen wie auch durch umfassendes Zusammentragen geographischer Reiseberichte ein weltumspannendes Wissen und folgte dann 1820 einem Rufe als Professor der Geographie an die Universität Berlin. Er war damit der erste Inhaber eines akademischen Lehrstuhls für Geographie im deutschen Sprachgebiet. Er lehrte dieses Fach gleichzeitig auch an der Berliner Kriegsschule.

Durch seine umfangreichen Werke über Afrika, Asien u. a. wies Ritter der geographischen Lehre neue Wege. Eine ausgezeichnete zeichnerische Begabung führte ihn auch zur Kartographie; er befruchtete diese in hohem Maße, war Bearbeiter und Herausgeber großer, zu Berlin und Gotha erstellter Atlanten, und er erfreute sich der besten Verbindungen zu Gelehrten und Kartenerstellern in aller Welt. 